\title{
A gene-editing/complementation strategy for tissue-specific lignin reduction while preserving biomass yield
}

Hasi Yu ${ }^{1,2}$, Chang Liu' and Richard A. Dixon ${ }^{1,2^{*}}$ (1)

\begin{abstract}
Background: Lignification of secondary cell walls is a major factor conferring recalcitrance of lignocellulosic biomass to deconstruction for fuels and chemicals. Genetic modification can reduce lignin content and enhance saccharification efficiency, but usually at the cost of moderate-to-severe growth penalties. We have developed a method, using a single DNA construct that uses CRISPR-Cas9 gene editing to knock-out expression of an endogenous gene of lignin monomer biosynthesis while at the same time expressing a modified version of the gene's open reading frame that escapes cutting by the Cas9 system and complements the introduced mutation in a tissue-specific manner.

Results: Expressing the complementing open reading frame in vessels allows for the regeneration of Arabidopsis plants with reduced lignin, wild-type biomass yield, and up to fourfold enhancement of cell wall sugar yield per plant. The above phenotypes are seen in both homozygous and bi-allelic heterozygous $\mathrm{T1}$ lines, and are stable over at least four generations.

Conclusions: The method provides a rapid approach for generating reduced lignin trees or crops with one single transformation event, and, paired with a range of tissue-specific promoters, provides a general strategy for optimizing loss-of-function traits that are associated with growth penalties. This method should be applicable to any plant species in which transformation and gene editing are feasible and validated vessel-specific promoters are available.
\end{abstract}

Keywords: Saccharification, Lignin modification, Gene editing, CRISPR, Cinnamoyl CoA reductase (CCR1)

\section{Background}

Lignocellulose biomass is highly abundant, energy-rich, and is constructed of polymers with carbon skeletons suitable for biological funneling into a range of fuels and replacement chemicals to help drive the bio-economy [1-3]. The major component of lignocellulose is secondary cell walls made of cellulose and hemicellulosic polysaccharides, which are impregnated with lignin [4]. Lignins are aromatic heteropolymers, composed mainly of guaiacyl (G) and syringyl (S) units, derived from the

*Correspondence: Richard.Dixon@unt.edu

${ }^{1}$ BioDiscovery Institute and Department of Biological Sciences, University of North Texas, 1155 Union Circle \#311428, Denton, TX 76203, USA

Full list of author information is available at the end of the article monolignols coniferyl alcohol and sinapyl alcohol, respectively $[5,6]$. Much smaller amounts of $p$-hydroxyphenyl $(\mathrm{H})$ units derived from $p$-coumaryl alcohol are also often present. More recently, lignins composed exclusively of caffeyl alcohol (C-lignin) have been found in seed coats of some monocot and dicot species [5]. The physical properties of lignin provide strength for plants to stand upright and hydrophobicity to allow efficient water transport through the vascular system [7]. Lignin also acts as a primary, constitutive physical barrier against ingress of pathogens and attack by herbivores [7, 8]. At the same time, the protective chemical and physical properties of lignin present a major impediment to the downstream processing of lignocellulosic biomass for multiple applications $[1,5,9]$. 
The complexation of lignin to polysaccharides hinders the enzymatic hydrolysis of cell wall polysaccharides into simple sugars during bioprocessing $[10,11]$. Because of this, modification of lignin content and/or composition can improve the enzymatic saccharification of biomass [12-22]. However, this is often accompanied by unacceptable reductions in biomass and seed yield $[1,9,12,13$, $15,23]$.

Although the molecular mechanisms underlying lignin modification-induced biomass reductions are still somewhat unclear [23-26], efforts to rescue such defects through recovery of vessel cell integrity have proved successful in the model plant Arabidopsis thaliana. To this end, the artificial SECONDARY WALL NAC BINDING ELEMENT of the XYLEM CYSTEINE PROTEASE1 promoter (ProSNBE) has been developed [27]; this confers high expression in both protoxylem and meta-xylem vessels. Tissue-specific complementation of the cinnamoyl CoA reductase 1 ( $c c r 1$ ) mutation in the lignin pathway by expression of ProSNBE:CCR1 resulted in restoration of biomass yield, and a fourfold increase in total sugar yield when compared with wild-type plants [28]. This approach requires transformation of an identified mutant and can therefore be difficult to apply to most bioenergy crops that lack mutant populations. CRISPR/ Cas9 technology itself is, however, applicable to biomass crops [29, 30], but generation of lignin pathway mutants by CRISPR/Cas9 and re-transformation to complement in order to restore vessel integrity under a vessel-specific promoter requires removal of the Cas9 protein to avoid cutting of the transformed gene. Theoretically, this could be done by knock-in or replacing with a new vesselspecific promoter to drive the gene encoding the lignin synthesis enzyme. However, the efficiency of CRISPR/ Cas9-derived knock-in or replacement is currently very low, and several generations will be required to obtain genetically stable material, thereby limiting the use of this approach [31, 32].

Recent studies have attempted to address the problem of reducing lignin by CRISPR/Cas9-mediated gene editing while maintaining biomass. Approaches include development of a screening method to identify efficient sgRNAs in Arabidopsis which target the enzyme hydroxycinnamoyl CoA: shikimate hydroxycinnamoyl transferase (HCT) coupled with use of an interfascicular fiber-specific promoter, pNST3, to drive CAS9 [33], or using CRISPR/Cas9 to target the CCR gene in hybrid poplar by screening for combinations of a null and a haploinsufficient $C C R$ allele to obtain a balance between reduced lignin and normal biomass [34].

We here report a simple approach, tested in the model species $A$. thaliana, to promote sugar release by reducing lignin content with maintenance of biomass yield.
We generated non-functional CCR1 with the regular CRISPR/Cas9 system, but fused to the vector a ProSNBE promoter driving a version of CCR1 encoding the wildtype protein but with its codons modified to escape cutting of the transgene by the Cas9 system. This method can provide plants with reduced lignin and normal growth in the T1 generation with a single transformation event and no need for complex screening. The phenotype is stable and inheritable.

\section{Results \\ Design of a CRISPR/Cas9 system for lignin reduction in the fiber region}

Restoring the lignin content to the xylem vessels in lignin biosynthesis mutants is a proven strategy to maintain lignin reduction in fibers while preserving biomass yield [28, 35-37]. However, as outlined above, current implementations are time consuming and with limited application for many crops and trees.

We have a developed a method to generate lignin pathway knock-out mutants and restore the expression of the targeted gene in xylem vessels using one simple construct. The strategy combines targeting Cas9 to the wild-type gene and restoring expression to xylem vessels by co-expressing a version of the targeted open reading frame with altered codon usage to escape recognition by the sgRNA and thereby avoid cutting by the Cas9 system. Figure 1a shows the sgRNA designed to target the A. thaliana CCR1 gene at the sequence ACC GTC TGC GTC ACC GGA GCT in the first exon, encoding the amino acid sequence $\mathrm{T} \mathrm{V} \mathrm{C} \mathrm{V} \mathrm{T} \mathrm{G} \mathrm{A.} \mathrm{The} \mathrm{sequence} \mathrm{for}$ vessel-specific complementation driven by the SNBE promoter encodes the same amino acid sequence with the nucleotides changed to ACA GTT TGT GTT ACA GGA GCG (Fig. 1b). High-frequency off-target mutagenesis induced by CRISPR/Cas9 has been reported in human and mouse cells, but seldom with mismatches over 3 base pairs [38-40]. Furthermore, deep sequencing of a total of 178 off-target sites showed that multiplex targeting in A. thaliana is highly specific to on-target sites with no detectable off-target events [41]. In the present case, we introduced six mismatches into the CCR1-coding region, and tested 24 T1 transgenic plants to check for off-target editing in the artificial CCR1.

\section{Efficient creation of a null mutation via targeted deletion at the AtCCR1 locus}

Using the scheme in Fig. 1, we designed a sgRNA which can target the CCR1 gene, and introduced this sgRNA into $p$ Cambia1300-CRISPR/Cas9 to knock out CCR1. Next, we cloned the CCR1-coding sequence into a T-vector harboring the SNBE promoter, and used a pointmutation kit to change the sequence to avoid targeting 
a

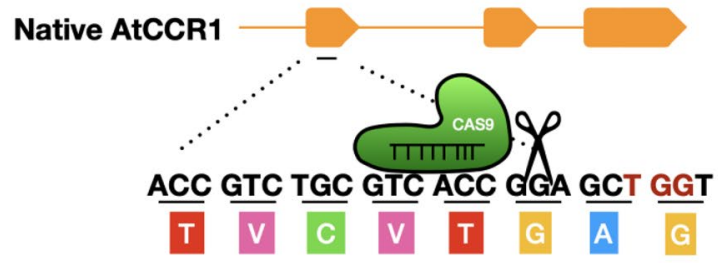

b

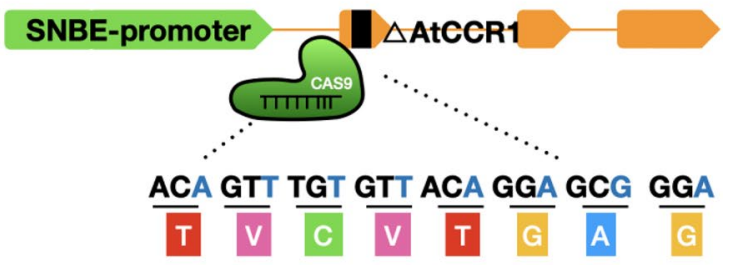

C

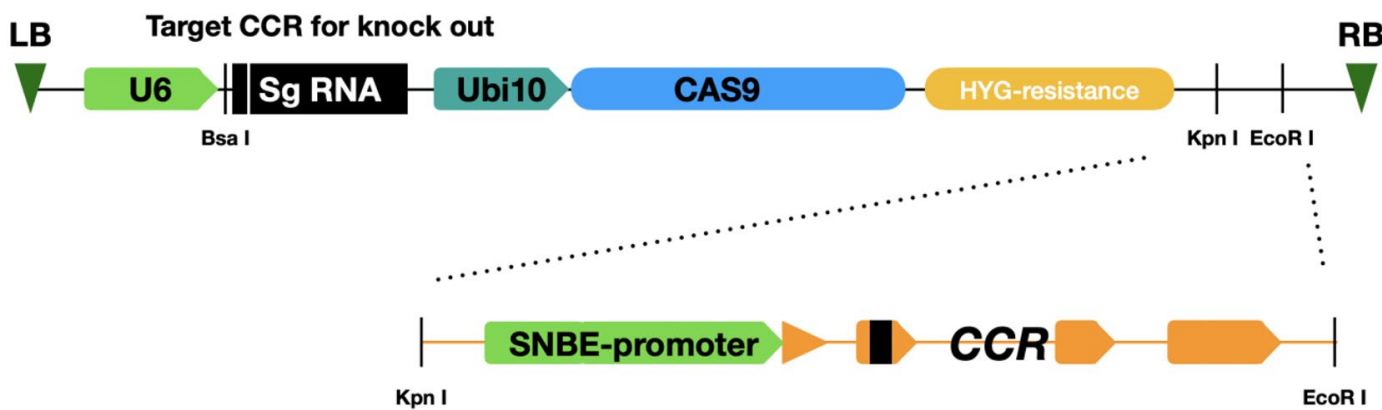

Fig. 1 Illustration of the strategy for reducing lignin content by targeting the AtCCR1 locus using a sgRNA/Cas9 design while restoring CCR1 function to vascular tissues. a Diagram of CRISPR/Cas9 system with a sgRNA that targets Cas9 to the native CCR1 gene. b Construct for vessel-specific expression of codon-modified CCR1 that encodes the same protein as native CCR1, driven by the xylem vessel-specific promoter SNBE. c Final construct for inserting the ProSNBE: artificial CCR1 into the pCambia 1300-CAS9 vector with the sgRNA designed for targeting the native CCR1

by the sgRNA as outlined above. Finally, we inserted ProSNBE: $\triangle A t C C R 1$ into the pCambia1300-CRISPR/ Cas 9 vector with endonucleases Kpn1 and EcoR1 (Fig. 1c).

One hundred and ninety-two independent T1 plants transformed with pCambia1300-CRISPR/Cas9ProSNBE: $\triangle A t C C R 1$ constructs were genotyped by Sanger sequencing of the target region. The sequencing results were analyzed by Tracking of Indels by Decomposition (TIDE), a tool which identifies the types and frequency of mutations in the region around the projected editing site [42]. The genotypes of the $\mathrm{T} 1$ plants are summarized in Fig. 2a. We found that 57/192 T1 plants ( $30 \%)$ were wild type, with no mutation detected in the endogenous CCR1 gene; 65/192 ( 34\%) were chimeric, namely wild type combined with more than two kinds of mutation in the target region; 19/192 ( 10\%) were heterozygous mutants, namely wild type combined with 1 kind of mutation in the target region; 34/192 ( 18\%) had no wild-type peak but two (bi-allelic) or more than two (tri-allelic) mutations; and 17/192 T1 plants ( 9\%) were homozygous mutants.

\section{Growth phenotype of T1 plants with homozygous CCR1 mutation}

We randomly selected six seedlings (genotypes shown in Fig. 2b) from the 17 homozygous mutants for further analysis; these represented one single nucleotide insertion and five deletions of 1, 2, 4 or 5 nucleotides. All these mutations changed the reading frame of CCR1, and disrupted gene function by creating premature termination codons at the start of the CCR $1 \mathrm{~N}$-terminus (Additional file 1: Fig. S1). We took the basal $5 \mathrm{~cm}$ of the stems of 12-week T1 plants for examination of cross-sections to visualize lignin deposition, and the remainder of the main stem for lignin measurement and sugar release assay after harvesting the $\mathrm{T} 1$ seeds (Fig. 2c).

Two-week-old T1 seedlings with homozygous Crisprmediated CCR1 mutation with co-expression of $\triangle C C R 1$ did not show the distinctive $c c r 1$ phenotype of small rosettes and shrunken leaves (Fig. $3 \mathrm{a}$ ); the growth phenotype was the same as wild type. An sgRNA targeting the first exon of CCR1 should generate a strong allele, so we selected $c c r 1-3$, a strong allele [9], as an additional control, and transformed it with ProSNBE:CCR1 as a check for the vessel-specific complemented phenotype. The growth characteristics of the ccr1-3/ ProSNBE:CCR1 and Crispr:CCR1:ProSNBE: $\triangle C C R 1$ transgenic lines appeared identical (Fig. 3C, D). Furthermore, T2-generation homozygous Crispr:CCR1:ProSNBE: $\triangle C C R 1$ edited lines that had lost the Crispr:CCR1:ProSNBE: $\triangle C C R 1$ construct showed a strong Crispr-generated $c c r 1$-negative growth 


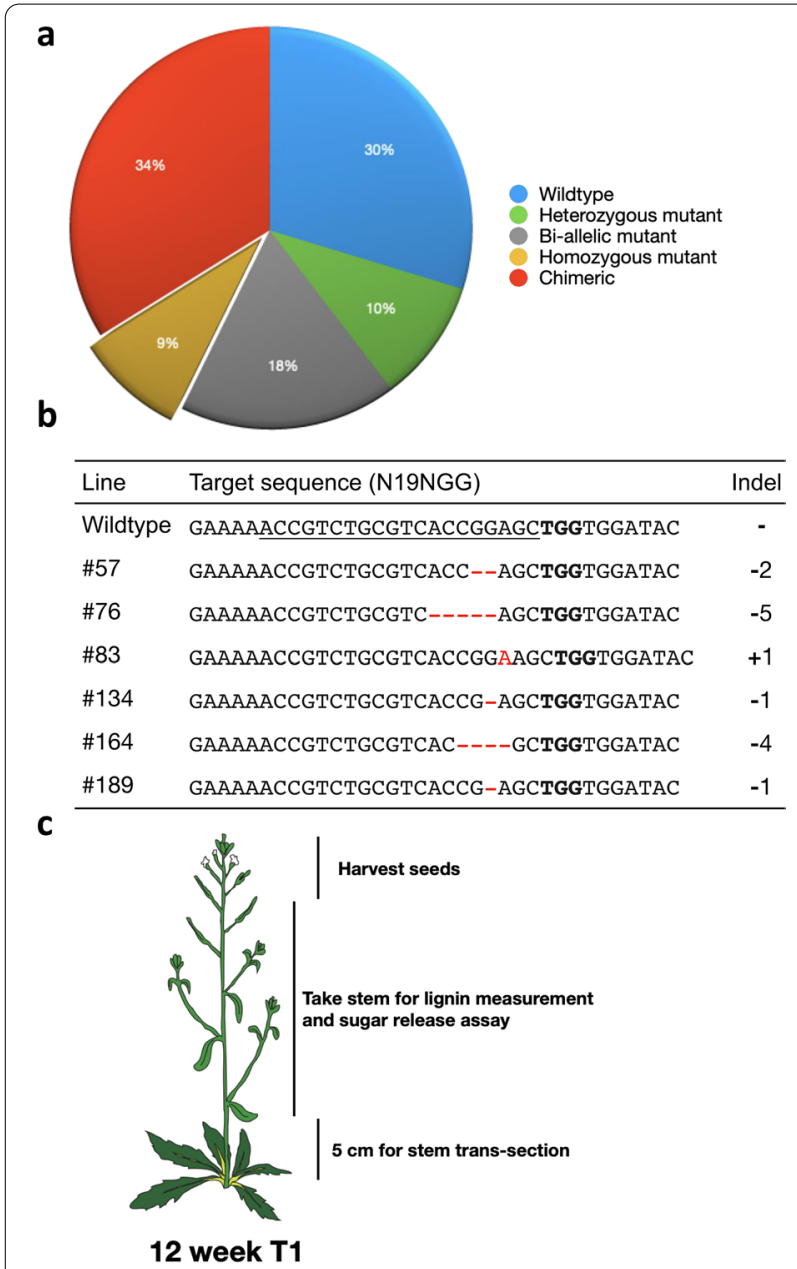

Fig. 2 Genotyping of T1 transgenic plants and the distribution of zygosity. a Zygosity of T1-edited plants. Following isolation of leaf DNA, genomic sequences spanning the sgRNA target site were PCR amplified and analyzed by Sanger sequencing. Transgenic plants of each line were classified based on mutation rate at the projected target site. $\mathbf{b}$ Target sequences of wild-type Col-0 and representative homozygous ccr 1 mutants in Cas9 transgenic lines. Their detection frequencies and the indel patterns (red color) are shown, and the sgRNA (underlined) and protospacer adjacent motif (PAM; bold text) sequences are highlighted. c Analysis scheme for gene-edited lines. For 12-week-old T1 homozygous and bi-allele ccr1 mutants, seeds were harvested from individual seedlings, the bottom $5 \mathrm{~cm}$ of inflorescence stems were taken for imaging of cross-sections for lignin deposition, and the main regions of inflorescence stems were harvested for lignin measurement and sugar release assay

phenotype, and growth rescue relied on the presence of Crispr:CCR1:ProSNBE: $\triangle C C R 1$ (Additional file 1: Fig. S2).

For further analysis, we transferred the T1 seedlings to larger pots, grew the plants for another 8 weeks (Fig. 3b), then measured the primary inflorescence stem height and the total weight of the Col-0, ccr1-3, ccr1-3
ProSNBE:CCR1 and Crispr:CCR1:ProSNBE: $\triangle C C R 1$ T1 lines. The height of the primary inflorescence stem of Crispr:CCR1:ProSNBE: $\triangle C C R 1$ T1 lines was equal to that of the wild type and $c c r 1-3$ ProSNBE:CCR1 lines, nearly twofold higher than that of the ccr1-3 T-DNA insertion mutant (Fig. 3b, c). Likewise, the total stem weight of ccr1 ProSNBE:CCR1 and Crispr:CCR1:ProSNBE: $\triangle C C R 1$ T1 lines was equal to that of the wild type, approximately twofold higher than that of the ccrl T-DNA insertion mutants (Fig. 3c). Homozygous lines of Crispr:CCR1:ProSNBE: $\triangle$ CCR1 exhibited a lodging phenotype, but some individuals among the bi-allele or weak mutants in CCR1 showed less lodging (but also less lignin reduction, see below).

\section{Crispr:CCR 1:ProSNBE: $\triangle C C R 1$ T1 lines show reduced lignin levels and improved saccharification efficiency}

The xylem tissue in wild-type $A$. thaliana plants contains large, open vessels, and the interfascicular fibers are massively lignified (Fig. 2d). In contrast, the xylem tissues in ccr1-3 mutants show an overall reduction in lignin deposition, as observed by both UV autofluorescence and Wiesner (phloroglucinol) staining, and develop irregularly shaped and collapsed vessels $[12,43,44]$. The xylem vessels of Crispr:CCR1:ProSNBE: $\triangle C C R 1 \mathrm{~T} 1$ lines showed an intense coloration on Wiesner staining and contained large open vessels similar to those of the wild type and the ccr1-3 ProSNBE:CCR1 lines [28] (Fig. 4d). The xylary fibers also appeared to be lignified. However, the interfascicular fibers showed reduced lignin deposition similar to the $c c r 1-3$ mutant line.

To examine the lignin content of stems of Crispr:CCR1:ProSNBE: $\triangle C C R 1$ T1 lines, we utilized the acetyl bromide spectrophotometric method [45, 46] for estimation of total lignin and the thioacidolysis method $[47,48]$ to reveal lignin monomer composition. The strong reduction in total lignin level observed in the $c c r 1-3$ mutant was partially rescued in the $c c r 1-3$ ProSNBE:CCR1 line and in some, but not all, of the Crispr:CCR1:ProSNBE: $\triangle C C R 1$ T1 lines (Fig. 4a). However, total thioacidolysis yields $(\mathrm{H}+\mathrm{G}+\mathrm{S})$ were strongly reduced in all lines compared to wild type (Fig. 4b). Larger effects on thioacidolysis yield compared to acetyl bromide lignin level are commonly seen in lignin genetic modification studies $[28,33,49]$. The two methods measure different features of the bulk lignin, with thioacidolysis only releasing $\beta$-O-4-linked monomer units [50, 51]. Our data are consistent with the lack of a significant increase in bulk lignin on vessel complementation of the A. thaliana ccr1-6 mutant allele as previously reported [28].

$\mathrm{H}$ lignin monomers constituted less than $2 \%$ of the total thioacidolysis-released monomer unit in wild-type 
a

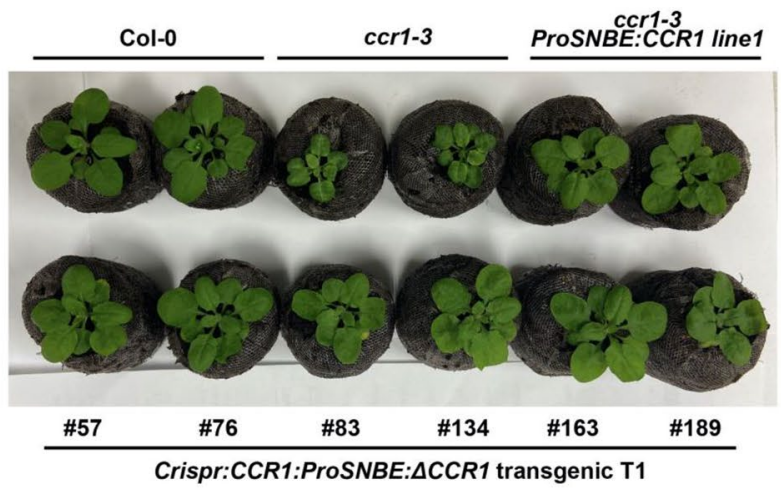

b

C
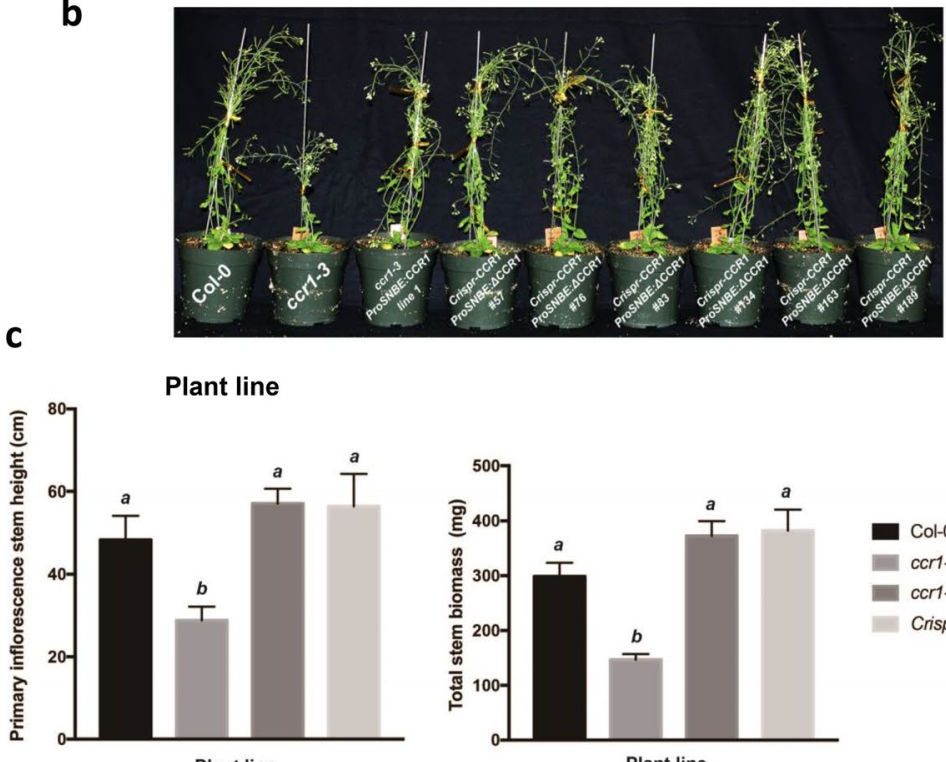

Col-0

acr1-3

ccr1-3 ProSNBE:CCR1 line1

Crispr:CCR1:ProSNBE:ACCR1 transgenic T1

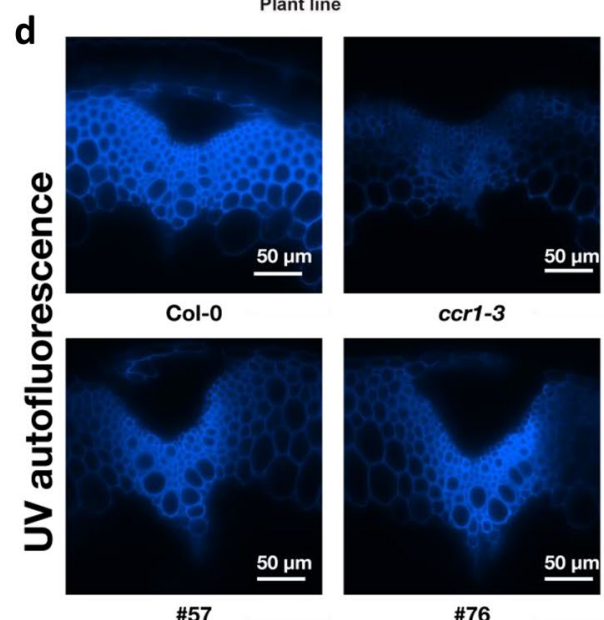

\#57 \#76

Crispr:CCR1:ProSNBE: $\Delta$ CCR1 Transgenic T1
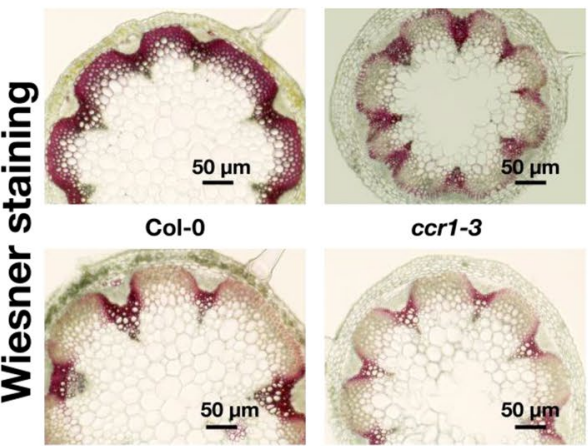

ccr1-3

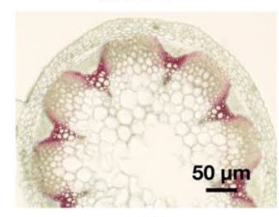

\#57

\#76

Crispr:CCR1:ProSNBE:ACCR1 Transgenic T1

Fig. 3 Phenotyping of T1 transgenic plants. a Phenotypes of 4 weeks Col-0, ccr1-3, ccr1-3 ProSNBE:CCR1 and transgenic

Crispr:CCR1:ProSNBE: $\triangle C C R 1 T 1$ lines. $\mathbf{b}$ Phenotypes of 12-week-old plants of the above lines. $\mathbf{c}$ Primary inflorescence stem height and biomass measurements of 12-week-old plants of the above lines. $\mathbf{d}$ Lignin deposition in inflorescence stems of the above lines. Transverse stem sections are shown with lignin autofluorescence and Wiesner staining. See Additional file 1: Fig. S4 for photographs of additional T1 plants. Bars $=200 \mu \mathrm{m}$. In $\mathbf{c}$, bars represent means and standard deviations, $n=6$. Letters above bars indicate statistical significance by one-way ANOVA, $p<0.05$ 


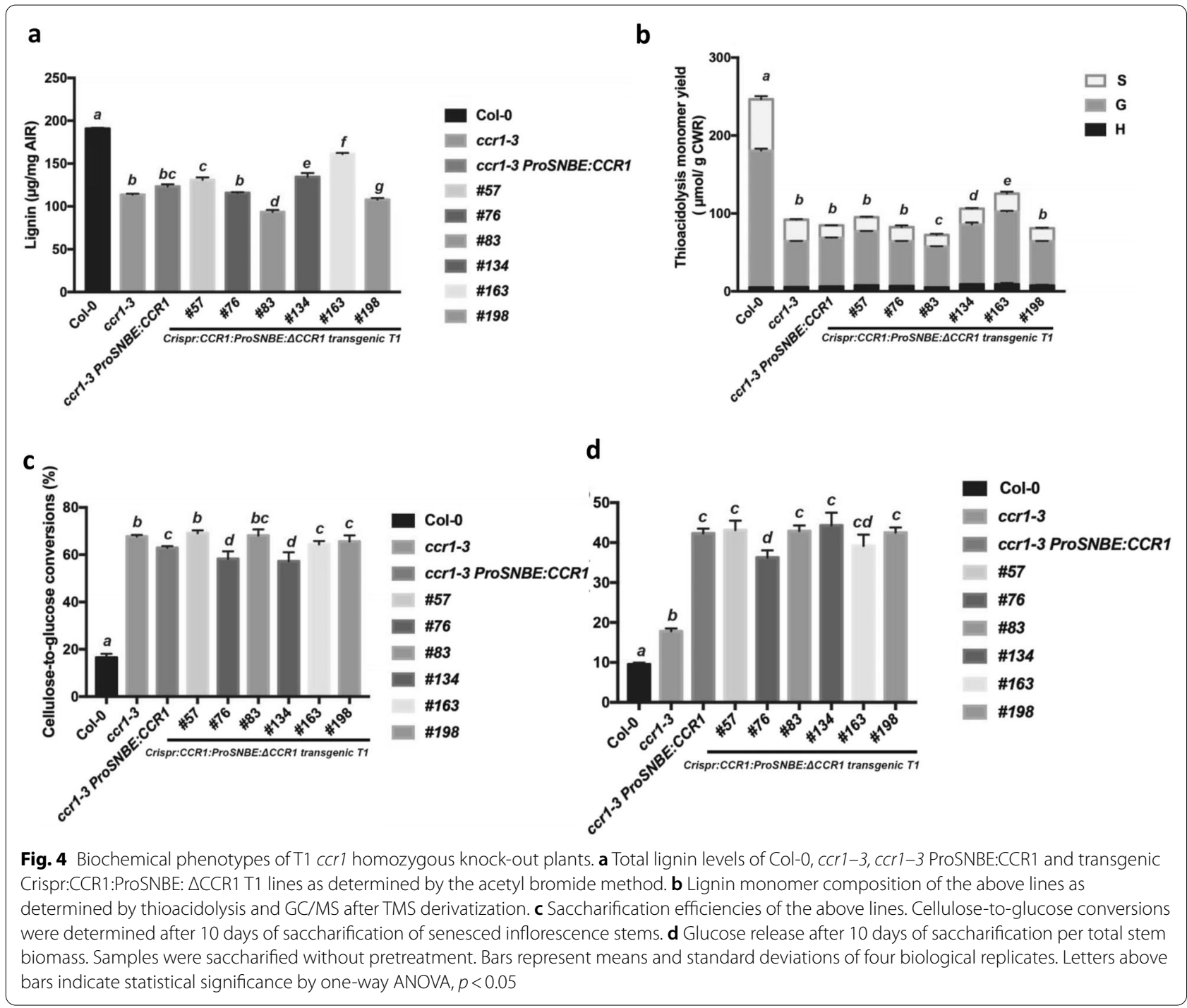

plants (Additional file 1: Fig. S3). $\mathrm{H}$ lignin content increased in ccr1-3, ccr1-3 ProSNBE:CCR1, and Crispr:CCR1:ProSNBE: $\triangle C C R 1$ T1. Furthermore, the $\mathrm{S} / \mathrm{G}$ ratio decreased in the ccrl-3 mutant compared to the wild type, and this decrease was maintained in the Crispr:CCR1:ProSNBE: $\triangle C C R 1 \mathrm{~T} 1$ plant as well as the ccr1-3 ProSNBE:CCR1 lines (Additional file 1: Fig. S3).

We next compared the saccharification efficiency without pretreatment for wild-type, ccr1-3, ccr1-3 ProSNBE:CCR1 and Crispr:CCR1:ProSNBE: $\triangle C C R 1$ $\mathrm{T} 1$ lines, using the upper portions of the main stems. The cellulose-to-glucose conversion for Crispr:CCR1:ProSNBE: $\triangle C C R 1$ T1 was approximately threefold higher than that of wild-type stems, similar to that of the $c c r 1-3$ mutant and the $c c r 1-3$ ProSNBE:CCR1 lines (Fig. 4c). The beneficial effect of the growth restoration arising from vessel-specific complementation can be seen in the total yields of sugar release per plant, which were over fourfold compared to only approximately twofold in the ccr1-3 mutants (Fig. 4d).

\section{Lignin reduction and improved saccharification efficiency are heritable in Crispr:CCR1:ProSNBE: $\triangle C C R 1$ lines}

Posttranscriptional gene silencing by RNA interference has been used for development of commercial low-lignin germplasm [52]. However, RNAi is not always heritable in plants [53]. Furthermore, RNAi of plant transgenes can sometimes occur without a trigger, as a stochastic phenomenon that shows limited epigenetic inheritance [54]. Therefore, there are advantages to avoiding RNAi approaches for fiber-specific targeting of lignin for biomass improvement.

Using a fiber-specific promoter to drive CAS9 expression with a sgRNA efficiently targeted the $A$. thaliana 
$H C T$ gene to reduce lignin content in fibers [33]. However, the T1 plants were chimeras, and it was not determined whether the favorable phenotype was stable and inheritable. Our approach generates homozygous mutations in the T1 generation, and the xylem vessels rescue relies on a linked transgene, suggesting that lines homozygous for the Crispr:CCR1:ProSNBE: $C C C R 1$ locus should provide stable lignin reduction without biomass loss.

To test this, we first identified single insertion lines among Crispr:CCR1:ProSNBE:ACCR1 T1 plants, using qPCR of the Cas 9 sequence to determine transgene copy number [55]. Using the $F L C$ gene as an endogenous homozygous single gene control, we found that lines $\# 57$ and \#83 possessed single heterozygous transgene insertions (Fig. 5a). To select homozygous Crispr:CCR1:ProSNBE: $\triangle C C R 1$ lines, seed from T1 generation \#57 and \#83 T1 seeds were collected and sown into soil. The T2 progeny showed segregation of wildtype and ccr1-like phenotypes. After genotyping, we found that all seedlings with the $c c r 1-3$ phenotype had lost the Crispr:CCR1:ProSNBE: $\triangle C C R 1$ transgene. $\mathrm{qPCR}$ analysis confirmed that Crispr:CCR1:ProSNBE: $\triangle C C R 1$ lines 83-T2-2, 83-T2-4, 83-T2-7, and 83-T2-11 were

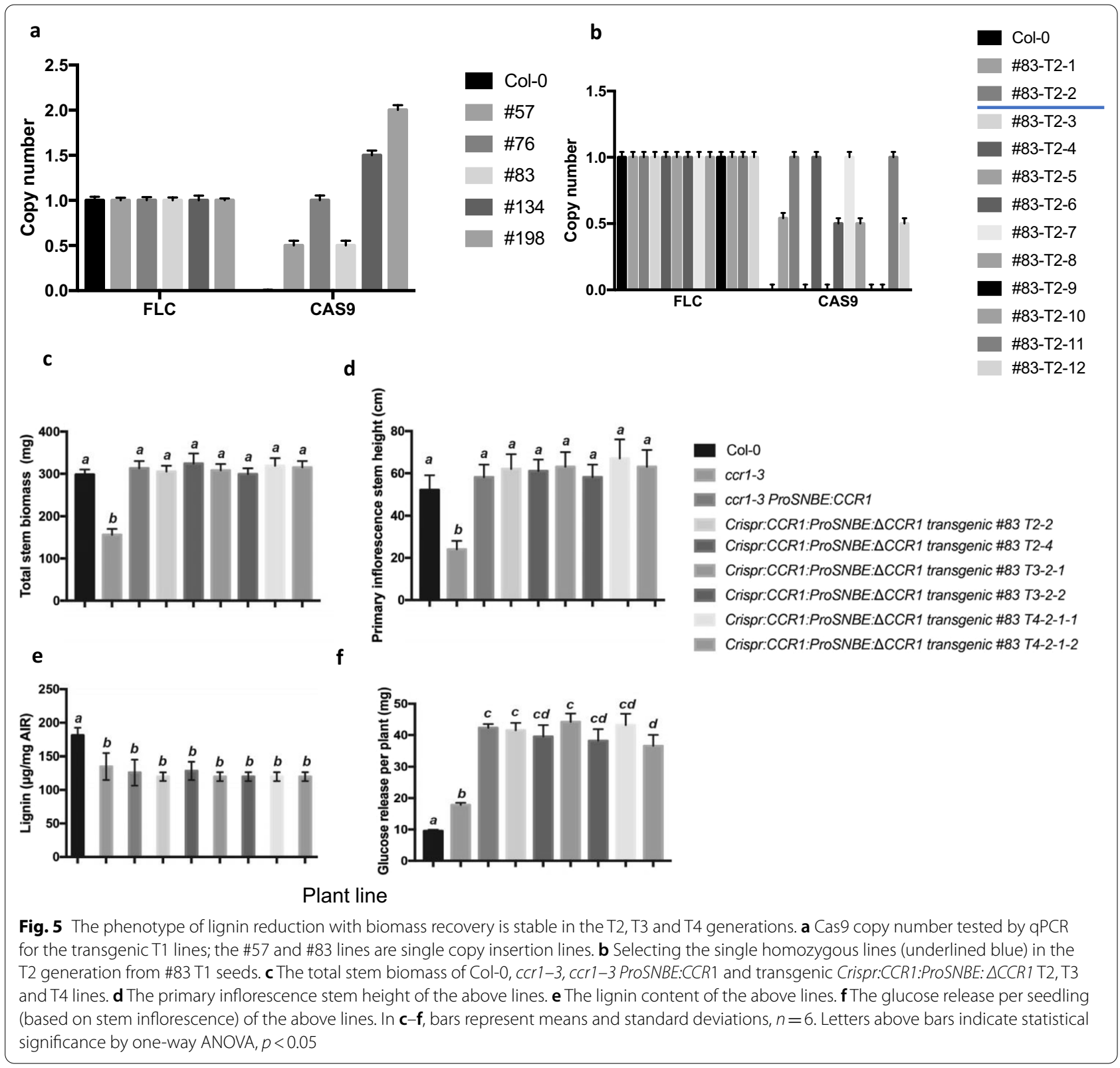


homozygous (Fig. 5b). After 12 weeks growth their seeds were collected, stem length and weight measured, and stem material stored at $-80{ }^{\circ} \mathrm{C}$ for further analysis. Seeds of line 83-T2-2 were continued to T4, and material collected after 12 weeks as before. The stem height and weight of the Crispr:CCR1:ProSNBE: $\triangle C C R 1$ lines was stable through the T4 generation (Fig. $5 \mathrm{c}$, d). Likewise, the decrease in acetyl bromide lignin content was stable through four generations at around the same level as observed in the ccr1-3 mutant (Fig. 5e), with a corresponding stable increase in saccharification measured as cell wall glucose equivalents released per plant (Fig. 5f).

\section{Bi-allelic $\mathrm{T} 1$ lines also show lignin reduction and high glucose yield without biomass loss}

Eighteen \% of the Crispr:CCR1:ProSNBE: $\triangle C C R 1$ $\mathrm{T} 1$ lines we generated were bi-allelic, meaning that although the lines may not be homozygous, the wildtype version of CCR1 is not present, suggesting that such lines may behave similarly to homozygous Crispr:CCR1:ProSNBE: $\triangle C C R 1$ transgenic lines. To test this hypothesis, we measured the primary stem height, total stem weight, lignin content, and glucose yield per plant. The stem weight and size of bi-allelic T1 plants was the same as wild type (Fig. 6a, b), the average lignin level the same as in the ccrl-3 mutant (Fig. 6c), and, most importantly, the average glucose release was about four times higher than wild type and two times higher than in ccr1-3 (Fig. 6d).

\section{Discussion}

In hybrid poplar, CCR down-regulation has been shown to yield up to $161 \%$ improvement in ethanol yield per unit of biomass [56]. However, the strong CCR RNAi downregulated poplar lines exhibit large biomass reductions. Suppressing lignin biosynthesis specifically in the fiber region can maintain growth while providing more sugar release in poplar [57]. The present method would provide a quick approach to test the best transgenes for improving saccharification efficiency in a tree or crop species assuming that vessel complementation could overcome the negative growth phenotype. Both CRISPR-gene editing and a suitable vessel-specific promoter are available in poplar, for example $[29,57]$. The method could also be used more generally in model, and potentially crop species, to determine, in cases where constitutive gene knock-down or knock-out results in yield penalties, which tissue requires wild-type expression of the targeted gene; in this case, the codon-modified transgene would have to be tested with a range of tissue-specific promoters known to be functional in the chosen species.

Theoretically, the same results might be obtained by direct use of a fiber-specific promoter to drive an antisense or hairpin sequence that targets the lignin biosynthesis gene. However, this may not provide a stable phenotype, especially after several generations, which is a drawback of the antisense and RNAi system [53].

Model species such as Arabidopsis and Medicago with mutant collections that cover most of the genome would not require the CRISPR component of the present strategy, and tissue-specific complementation in such cases is already established [28]. However, for most crops and trees such mutant resources are not available, and complementing existing gene-edited lines would require removal of the Cas 9 and a second round of transformation to provide the vessel-specific expression with an experimentally validated promoter. The method described here should prove time-saving for basic studies in crops. Its limitation for commercial application, however, is that the Cas9 and the complementing vessel-specific transcript are on the same construct which, although simplifying development of homozygous progeny lines, means that is not possible to segregate out the Cas9 in subsequent generations. Furthermore, strong lignin down-regulation in fibers can lead to lodging, as in the present case, and it will therefore be necessary to balance improved processing ability with agronomic performance in selecting lines for further trait development.

\section{Conclusions}

In conclusion, we have developed a simple method to obtain, in one generation after a single transformation event, plants with reduced lignin level and higher cell wall glucose release per plant, with no accompanying reduction in biomass yield. In our experiments with $A$. thaliana, 27\% of T1 plants showed this advanced phenotype, we could quickly get homozygous transgenic plants with simple genotyping, and the phenotype was stable and inheritable. Further experiments will be necessary to show the utility of this approach in other diploid species, and whether it can be readily translated to forage or bioenergy crops such as alfalfa (Medicago sativa) and switchgrass (Panicum virgatum) with more complex ploidy levels. As presently formulated, the method is advantageous for basic studies, but approaches that allow for removal of the Cas9 may be necessary to facilitate deregulation of transgenic lines for commercial application.

\section{Methods}

\section{Plant growth and transformation}

Arabidopsis thaliana plants were germinated on $1 / 2$ Murashige and Skoog (MS) agar medium with sucrose $(15 \mathrm{~g} / \mathrm{L})$ at $4{ }^{\circ} \mathrm{C}$ for 3 days and transferred to a growth chamber at $22{ }^{\circ} \mathrm{C}$ with a photoperiod of $16 \mathrm{~h}$ light $/ 8 \mathrm{~h}$ 

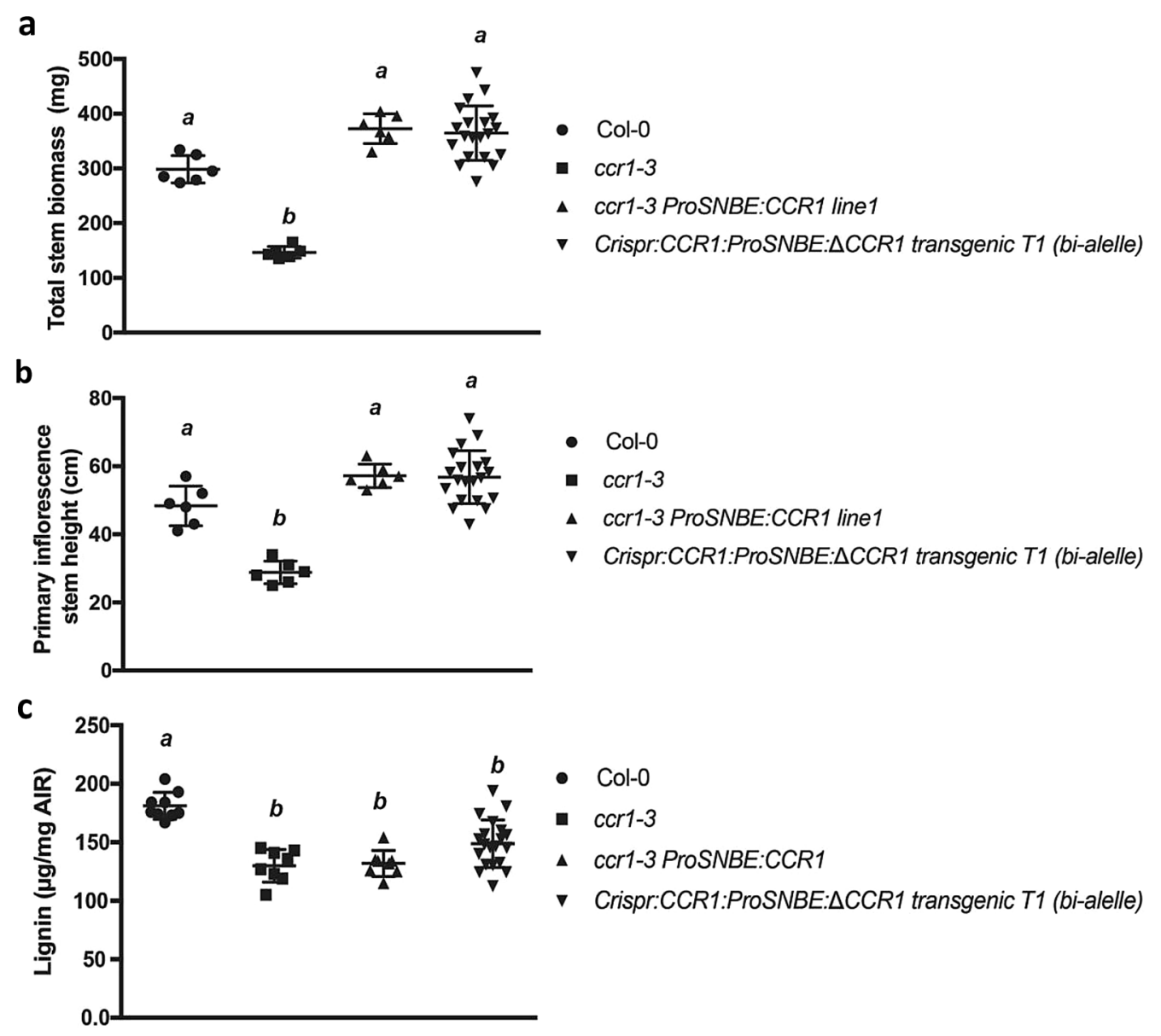

Col-0

- ccr1-3

- ccr1-3 ProSNBE:CCR1

- Crispr:CCR1:ProSNBE:ACCR1 transgenic T1 (bi-alelle)

d

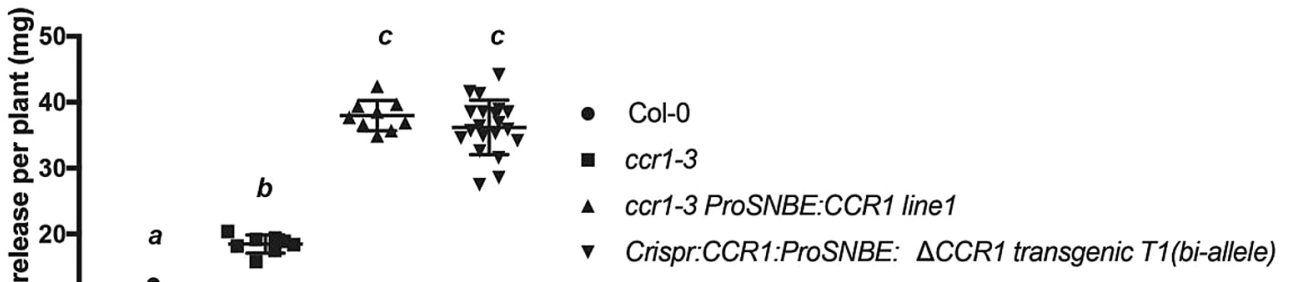

Fig. 6 The lignin reduction and biomass recovery phenotype can be detected in T1 bi-allele plants. a The total stem biomass of Col-0, ccr1-3, cCr1-3 ProSNBE:CCR1 and transgenic Crispr:CCR1:ProSNBE: $\triangle C C R 1$ bi-allele lines. $\mathbf{b}$ The primary inflorescence stem height of the above lines. $\mathbf{c}$ The lignin content of the above lines. $\mathbf{d}$ The glucose release per seedling of the above lines. Data points show individual plants. Bars represent means and standard errors

dark for 2 weeks. Seedlings were then transferred to soil and growth continued under the same conditions. Transformation of $A$. thaliana was performed by the floral dip method [58]. Transgenic plants were isolated on $1 / 2 \mathrm{MS}$ medium containing $50 \mu \mathrm{g} / \mathrm{mL}$ hygromycin, and positive seedlings transfer to soil "cookies". Positive T1 transgenic plants were used for further analysis.

\section{Gene cloning and plasmid construction}

The guide RNA (sgRNA) was designed with Geneious R7 to target the first exon of CCR1, and cloned into pCambial 300-35S-Cas9 after the AtU6 promoter [59]. The CCR1 open reading frame was PCR amplified from Col-0 cDNA and cloned into pMD20 vector (Takara). The artificial SNBE promoter contained three copies of XCP1SNBE1 fused to the CaMV 35S minimal promoter (from 
246 to 21 ), as described previously [60]. To construct this synthetic promoter, a 103-bp construct containing the restriction sites for $\mathrm{XbaI}$ and $\mathrm{NdeI}$ was first synthesized as a long primer by Invitrogen (Life Technologies). After dilution to $20 \mathrm{nM}$, this was mixed with $5 \mu \mathrm{L}$ each of forward and reverse primers (Additional file 1: Table S1), followed by annealing with PCR, down from 95 to $25^{\circ} \mathrm{C}$ at $0.1{ }^{\circ} \mathrm{C}$ per second. The artificial SNBE promoter was cloned into the XbaI- and NdeI-digested pMD20 T-vector containing the AtCCR1 coding sequence using T4 ligase. Mutations at the sgRNA target site of AtCCR1 were generated using pMD20 containing ProSNBE$A t C C R$ with a Site-Directed Mutagenesis Kit (NEB, Cat. \# E0554S), to make the artificial CCR1 with wild-type amino acid sequence but altered codons to avoid cutting by the CRISPR system. ProSNBE: $\triangle A t C C R 1$ was PCR amplified and cloned into pCambia1300-35S-Cas9 with the sgRNA targeting native CCR1 using KpnI and EcoI.

\section{Genotyping}

For Sanger sequencing, DNA was extracted from leaves 3 weeks after planting. Amplification used a forward PCR primer $500 \mathrm{bp}$ upstream of the sgRNA target site, located on the native CCR1 promoter, and a reverse primer $400 \mathrm{bp}$ downstream of the sgRNA target site. The native CCR 1 can be isolated by these 2 primers but the transgenic CCR1 cannot. To obtain better sequencing signal, an independent primer $400 \mathrm{bp}$ upstream of the sgRNA target site was used for sequencing. All primers are listed in Additional file 1: Table S1. The sequencing results were uploaded and analyzed for mutation rate with TIDE (https://tide.deskgen.com/) [42], using wild-type samples of CCR1 as the reference. The homozygous and bi-allele mutant results were double checked with Geneious R7.

\section{Microscopy analyses}

The basal internodes of inflorescence stems of 12-weekold plants were collected. Free-hand cross sections of A. thaliana inflorescence stems were stained with $0.5 \%$ phloroglucinol (w/v) (Sigma-Aldrich) in 12\% (v/v) $\mathrm{HCl}$ for $3 \mathrm{~min}$, and immediately observed under a bright-field and UV-B microscope.

\section{Lignin analyses}

Mature inflorescence stems from independent 12-weekold $A$. thaliana Col-0, ccr1 mutant and transgenic plants were harvested after collecting seeds and ground in liquid nitrogen to a fine powder to prepare the alcohol-insoluble residue (AIR) as previously described [46]. After de-starching [61], the crystalline cellulose content and monosaccharide composition were analyzed according to a previously published protocol [62]. The lignin content was determined as described [46]. Lignin composition was determined by an optimized thioacidolysis method [63]. Approximately $2 \mathrm{mg}$ of dry stem material was used for analysis per replicate, and four biological replicates were analyzed.

\section{Saccharification assays}

Inflorescence stems were collected after harvesting seeds, chopped into $2-4 \mathrm{~mm}$ pieces, $10-\mathrm{mg}$ stem samples placed into 2-mL Eppendorf tube with steel beads, and the tissue ground at low temperature. Cell wall residue (CWR) was obtained by heating the crushed samples in water at $98{ }^{\circ} \mathrm{C}$, moving the residues into 2 - $\mathrm{mL}$ glass vials, extracting sequentially with ethanol, chloroform, and acetone, and drying the remaining CWR under vacuum. The amount of cellulose in the CWR was estimated colorimetrically [9]. Saccharification assays (without pretreatment) were performed as described previously, with a 10-day incubation period and enzyme added every 2 days. To estimate the amount of glucose released, the glucose oxidase (GOD)-peroxidase (POD) method was used [64].

\section{Supplementary Information}

The online version contains supplementary material available at https://doi. org/10.1186/s13068-021-02026-5.

Additional file 1: Figure S1. Recovered mutations in native

CCR1 loci. Figure S2. The growth rescue relies on the presence of Crispr:CCR1:ProSNBE: $\triangle C C R 1$. Figure S3. Lignin composition in wild-type, cCr1-3, ccr1-3 ProSNBE:CCR1 and Crispr:CCR1:ProSNBE: $\triangle C C R 1$ T1 plants. Figure S4. Lignin deposition patterns in inflorescence stems of T1 transgenic plants. Table S1. Primers used in the present work.

\section{Acknowledgements}

We thank Dr. Yanfei Mao, Shanghai Center for Plant Stress Biology (PSC), for providing the pCambia1300-Cas9 vector, and Dr. Wout Boerjan, VIB Gent, for providing the ProSNBE:CCR1 control plants.

\section{Authors' contributions}

$\mathrm{HY}$ and RAD conceived the research. $\mathrm{HY}$ and $\mathrm{CL}$ performed experiments and analyzed data. RAD wrote the manuscript. All authors read and approved the final manuscript.

\section{Funding}

This work was supported by the Center for Bioenergy Innovation (Oak Ridge National Laboratory), a US Department of Energy (DOE) Bioenergy Research Center supported by the Office of Biological and Environmental Research in the DOE Office of Science.

Availability of data and materials

The data supporting the conclusions of this article are included within the article and its additional files.

\section{Declarations}

Ethics approval and consent to participate Not applicable.

Consent for publication

Not applicable. 


\section{Competing interests}

The authors declare they have no conflict of interest.

\section{Author details}

${ }^{1}$ BioDiscovery Institute and Department of Biological Sciences, University of North Texas, 1155 Union Circle \#311428, Denton, TX 76203, USA. ${ }^{2}$ Center for Bioenergy Innovation (CBI), Oak Ridge National Laboratory, Oak Ridge, TN 37831, USA.

Received: 19 June 2021 Accepted: 25 August 2021

Published online: 03 September 2021

\section{References}

1. Vanholme B, Desmet T, Ronsse F, Rabaey K, Van Breusegem F, De Mey $M$, Soetaert W, et al. Towards a carbon-negative sustainable bio-based economy. Front Plant Sci. 2013;4:174.

2. Isikgor FH, Becer CR. Lignocellulosic biomass: a sustainable platform for the production of bio-based chemicals and polymers. Polymer Chem. 2015;6:4497-559.

3. Mallinson SJ, Machovina MM, Silveira RL, Garcia-Borràs M, Gallup N, Johnson CW, Allen MD, et al. A promiscuous cytochrome P450 aromatic O-demethylase for lignin bioconversion. Nat Commun. 2018;9:1-12.

4. Cosgrove DJ. Growth of the plant cell wall. Nat Rev Mol Cell Biol. 2005:6:850-61.

5. Chen F, Tobimatsu Y, Havkin-Frenkel D, Dixon RA, Ralph J. A polymer of caffeyl alcohol in plant seeds. Proc Natl Acad Sci USA. 2012;109:1772-7.

6. Boerjan W, Ralph J, Baucher M. Lignin biosynthesis. Annu Rev Plant Biol. 2003;54:519-46.

7. Weng JK, Chapple C. The origin and evolution of lignin biosynthesis. New Phytol. 2010;187:273-85.

8. Miedes E, Vanholme R, Boerjan W, Molina A. The role of the secondary cell wall in plant resistance to pathogens. Front Plant Sci. 2014;5:358.

9. Van Acker R, Vanholme R, Storme V, Mortimer JC, Dupree P, Boerjan W. Lignin biosynthesis perturbations affect secondary cell wall composition and saccharification yield in Arabidopsis thaliana. Biotechnol Biofuels. 2013;6:1-17.

10. Weng J-K, Li X, Bonawitz ND, Chapple C. Emerging strategies of lignin engineering and degradation for cellulosic biofuel production. Curr Opin Biotechnol. 2008;19:166-72.

11. Pauly M, Keegstra K. Plant cell wall polymers as precursors for biofuels. Curr Opin Plant Biol. 2010;13:304-11.

12. Goujon T, Ferret V, Mila I, Pollet B, Ruel K, Burlat V, Joseleau J-P, et al. Down-regulation of the AtCCR1 gene in Arabidopsis thaliana: effects on phenotype, lignins and cell wall degradability. Planta. 2003;217:218-28.

13. Chen F, Dixon RA. Lignin modification improves fermentable sugar yields for biofuel production. Nat Biotechnol. 2007;25:759-61.

14. Leple J-C, Dauwe R, Morreel K, Storme V, Lapierre C, Pollet B, Naumann $A$, et al. Downregulation of cinnamoyl-coenzyme A reductase in poplar: multiple-level phenotyping reveals effects on cell wall polymer metabolism and structure. Plant Cell. 2007;19:3669-91.

15. Shadle G, Chen F, Reddy MS, Jackson L, Nakashima J, Dixon RA. Downregulation of hydroxycinnamoyl CoA: shikimate hydroxycinnamoyl transferase in transgenic alfalfa affects lignification, development and forage quality. Phytochemistry. 2007;68:1521-9.

16. Jackson LA, Shadle GL, Zhou R, Nakashima J, Chen F, Dixon RA. Improving saccharification efficiency of alfalfa stems through modification of the terminal stages of monolignol biosynthesis. Bioenergy Res. 2008;1:180-92.

17. Day A, Neutelings G, Nolin F, Grec S, Habrant A, Crônier D, Maher B, et al. Caffeoyl coenzyme A O-methyltransferase down-regulation is associated with modifications in lignin and cell-wall architecture in flax secondary xylem. Plant Physiol Biochem. 2009;47:9-19.

18. Voelker SL, Lachenbruch B, Meinzer FC, Jourdes M, Ki C, Patten AM, Davin $L B$, et al. Antisense down-regulation of $4 C L$ expression alters lignification, tree growth, and saccharification potential of field-grown poplar. Plant Physiol. 2010;154:874-86

19. Eudes A, George A, Mukerjee P, Kim JS, Pollet B, Benke PI, Yang F, et al. Biosynthesis and incorporation of side-chain-truncated lignin monomers to reduce lignin polymerization and enhance saccharification. Plant Biotechnol J. 2012;10:609-20.

20. Mansfield SD, Kang KY, Chapple C. Designed for deconstruction-poplar trees altered in cell wall lignification improve the efficacy of bioethanol production. New Phytol. 2012;194:91-101.

21. Mottiar Y, Vanholme R, Boerjan W, Ralph J, Mansfield SD. Designer lignins: harnessing the plasticity of lignification. Curr Opin Biotechnol. 2016;37:190-200

22. Wilkerson C, Mansfield S, Lu F, Withers S, Park J-Y, Karlen S, Gonzales-Vigil $\mathrm{E}$, et al. Monolignol ferulate transferase introduces chemically labile linkages into the lignin backbone. Science. 2014;344:90-3.

23. Bonawitz ND, Chapple C. Can genetic engineering of lignin deposition be accomplished without an unacceptable yield penalty? Curr Opin Biotechnol. 2013:24:336-43.

24. Carmona C, Langan P, Smith JC, Petridis L. Why genetic modification of lignin leads to low-recalcitrance biomass. Phys Chem Chem Phys. 2015;17:358-64.

25. Ha CM, Fine D, Bhatia A, Rao X, Martin MZ, Engle NL, Wherritt DJ, et al. Ectopic defense gene expression is associated with growth defects in Medicago truncatula lignin pathway mutants. Plant Physiol. 2019;181:63-84.

26. Muro-Villanueva F, Mao X, Chapple C. Linking phenylpropanoid metabolism, lignin deposition, and plant growth inhibition. Curr Opin Biotechnol. 2019;56:202-8

27. Zhong R, Lee C, Ye Z-H. Global analysis of direct targets of secondary wall NAC master switches in Arabidopsis. Mol Plant. 2010;3:1087-103.

28. De Meester B, de Vries L, Özparpucu M, Gierlinger N, Corneillie S, Pallidis A, Goeminne G, et al. Vessel-specific reintroduction of CINNAMOYL-COA REDUCTASE1 (CCR1) in dwarfed ccr1 mutants restores vessel and xylary fiber integrity and increases biomass. Plant Physiol. 2018;176:611-33.

29. An Y, Geng Y, Yao J, Fu C, Lu M, Wang C, Du J. Efficient genome editing in Populus using CRISPR/Cas12a. Front Plant Sci. 2020;11:593938.

30. Park JJ, Yoo CG, Flanagan A, Pu Y, Debnath S, Ge Y, Ragauskas AJ, Wang ZY. Defined tetra-allelic gene disruption of the 4-coumarate:coenzyme A ligase 1 (PV4CL1) gene by CRISPR/Cas9 in switchgrass results in lignin reduction and improved sugar release. Biotechnol Biofuels. 2017;10:284.

31. Fauser F, Roth N, Pacher M, llg G, Sánchez-Fernández R, Biesgen C, Puchta H. In planta gene targeting. Proc Natl Acad Sci USA. 2012;109:7535-40.

32. Miki D, Zhang W, Zeng W, Feng Z, Zhu J-K. CRISPR/Cas9-mediated gene targeting in Arabidopsis using sequential transformation. Nat Commun. 2018;9:1-9.

33. Liang $Y$, Eudes $A$, Yogiswara $S$, Jing B, Benites VT, Yamanaka $R$, Cheng-Yue $C$, et al. A screening method to identify efficient sgRNAs in Arabidopsis, used in conjunction with cell-specific lignin reduction. Biotechnol Biofuels. 2019:12:1-15.

34. De Meester B, Calderón BM, de Vries L, Pollier J, Goeminne G, Van Doorsselaere J, Chen M, et al. Tailoring poplar lignin without yield penalty by combining a null and haploinsufficient CINNAMOYL-COA REDUCTASE2 allele. Nat Commun. 2020;11:1-13.

35. Kubo M, Udagawa M, Nishikubo N, Horiguchi G, Yamaguchi M, Ito J, Mimura T, et al. Transcription switches for protoxylem and metaxylem vessel formation. Genes Dev. 2005;19:1855-60.

36. Zhong R, Lee C, Zhou J, McCarthy RL, Ye Z-H. A battery of transcription factors involved in the regulation of secondary cell wall biosynthesis in Arabidopsis. Plant Cell. 2008;20:2763-82.

37. Vargas L, Cesarino I, Vanholme R, Voorend W, Saleme MdLS, Morreel K, Boerjan W. Improving total saccharification yield of Arabidopsis plants by vessel-specific complementation of caffeoyl shikimate esterase (cse) mutants. Biotechnol Biofuels. 2016;9:1-16.

38. Fu Y, Foden JA, Khayter C, Maeder ML, Reyon D, Joung JK, Sander JD. High-frequency off-target mutagenesis induced by CRISPR-Cas nucleases in human cells. Nat Biotechnol. 2013:31:822-6.

39. Hsu PD, Scott DA, Weinstein JA, Ran FA, Konermann S, Agarwala V, Li $Y$, et al. DNA targeting specificity of RNA-guided Cas9 nucleases. Nat Biotechnol. 2013;31:827-32.

40. Aryal NK, Wasylishen AR, Lozano G. CRISPR/Cas9 can mediate highefficiency off-target mutations in mice in vivo. Cell Death Dis. 2018;9:1-3.

41. Peterson BA, Haak DC, Nishimura MT, Teixeira PJ, James SR, Dangl JL, Nimchuk ZL. Genome-wide assessment of efficiency and specificity in CRISPR/Cas9 mediated multiple site targeting in Arabidopsis. PLOS ONE. 2016:11:e0162169. 
42. Brinkman EK, Chen T, Amendola M, van Steensel B. Easy quantitative assessment of genome editing by sequence trace decomposition. Nucleic Acids Res. 2014;42:e168-e168.

43. Jones $L$, Ennos AR, Turner SR. Cloning and characterization of irregular xylem4 (irx4): a severely lignin-deficient mutant of Arabidopsis. Plant J. 2001;26:205-16.

44. Derikvand MM, Sierra JB, Ruel K, Pollet B, Do C-T, Thévenin J, Buffard D, et al. Redirection of the phenylpropanoid pathway to feruloyl malate in Arabidopsis mutants deficient for cinnamoyl-CoA reductase 1. Planta. 2008;227:943-56

45. Fukushima RS, Hatfield RD. Extraction and isolation of lignin for utilization as a standard to determine lignin concentration using the acetyl bromide spectrophotometric method. J Agric Food Chem. 2001;49:3133-9.

46. Foster CE, Martin TM, Pauly M. Comprehensive compositional analysis of plant cell walls (lignocellulosic biomass) part I: lignin. J Vis Exp. 2010; e1745.

47. Lapierre C, Pollet B, Rolando C. New insights into the molecular architecture of hardwood lignins by chemical degradative methods. Res Chem Intermed. 1995;21:397-412.

48. Chen F, Srinivasa Reddy MS, Temple S, Jackson L, Shadle G, Dixon RA. Multi-site genetic modulation of monolignol biosynthesis suggests new routes for formation of syringyl lignin and wall-bound ferulic acid in alfalfa (Medicago sativa L.). Plant J. 2006;48:113-24.

49. Zhao Q, Tobimatsu Y, Zhou R, Pattathil S, Gallego-Giraldo L, Fu C, Jackson LA, et al. Loss of function of cinnamyl alcohol dehydrogenase 1 leads to unconventional lignin and a temperature-sensitive growth defect in Medicago truncatula. Proc Natl Acad Sci USA. 2013;110:13660-5.

50. Lapierre C, Monties B, Rolando C, de Chirale L. Thioacidolysis of lignin: comparison with acidolysis. J Wood Chem Technol. 1985;5:277-92.

51. Hatfield R, Grabber J, Ralph J, Brei K. Using the acetyl bromide assay to determine lignin concentrations in herbaceous plants: some cautionary notes. J Agric Food Chem. 1999;47:628-32.

52. Barros J, Temple S, Dixon RA. Development and commercialization of reduced lignin alfalfa. Curr Opin Biotechnol. 2019;56:48-54.

53. Jones $L$, Ratcliff F, Baulcombe DC. RNA-directed transcriptional gene silencing in plants can be inherited independently of the RNA trigger and requires Met1 for maintenance. Curr Biol. 2001;11:747-57.

54. Kunz C, Schöb H, Stam M, Kooter JM, Meins F Jr. Developmentally regulated silencing and reactivation of tobacco chitinase transgene expression. Plant J. 1996;10:437-50.
55. Głowacka K, Kromdijk J, Leonelli L, Niyogi KK, Clemente TE, Long SP. An evaluation of new and established methods to determine T-DNA copy number and homozygosity in transgenic plants. Plant Cell Environ. 2016;39:908-17.

56. Van Acker R, Leplé J-C, Aerts D, Storme V, Goeminne G, Ivens B, Légée $F$, et al. Improved saccharification and ethanol yield from field-grown transgenic poplar deficient in cinnamoyl-CoA reductase. Proc Natl Acad Sci USA. 2014;111:845-50.

57. Gui J, Lam PY, Tobimatsu Y, Sun J, Huang C, Cao S, Zhong Y, et al. Fibrespecific regulation of lignin biosynthesis improves biomass quality in Populus. New Phytol. 2020;226:1074-87.

58. Clough SJ, Bent AF. Floral dip: a simplified method for Agrobacteriummediated transformation of Arabidopsis thaliana. Plant J. 1998;16:735-43.

59. Mao Y, Zhang H, Xu N, Zhang B, Gou F, Zhu J-K. Application of the CRISPR-Cas system for efficient genome engineering in plants. Mol Plant. 2013:6:2008-11.

60. MCCarthy RL, Zhong R, Ye Z-H. Secondary wall NAC binding element (SNBE), a key cis-acting element required for target gene activation by secondary wall NAC master switches. Plant Signal Behav. 2011;6:1282-5.

61. Foster CE, Martin TM, Pauly M. Comprehensive compositional analysis of plant cell walls (lignocellulosic biomass) part II: carbohydrates. J Vis Exp. 2010. https://doi.org/10.3791/1837.

62. Yu L, Chen H, Sun J, Li L. PtrKOR1 is required for secondary cell wall cellulose biosynthesis in Populus. Tree Physiol. 2014;34:1289-300.

63. Chen F, Zhuo C, Xiao X, Pendergast TH, Devos KM. A rapid thioacidolysis method for biomass lignin composition and tricin analysis. Biotechnol Biofuels. 2021;14:1-9.

64. Van Acker R, Vanholme R, Piens K, Boerjan W. Saccharification protocol for small-scale lignocellulosic biomass samples to test processing of cellulose into glucose. Bio-Protoc. 2016. https://doi.org/10.21769/BioProtoc. 1701.

\section{Publisher's Note}

Springer Nature remains neutral with regard to jurisdictional claims in published maps and institutional affiliations.
Ready to submit your research? Choose BMC and benefit from:

- fast, convenient online submission

- thorough peer review by experienced researchers in your field

- rapid publication on acceptance

- support for research data, including large and complex data types

- gold Open Access which fosters wider collaboration and increased citations

- maximum visibility for your research: over $100 \mathrm{M}$ website views per year

At BMC, research is always in progress.

Learn more biomedcentral.com/submissions 